\title{
Coherent light scattering by interacting anisotropic molecules with variable dipolar polarizability $\left(^{*}\right)$
}

\author{
S. Kielich \\ Nonlinear Optics Division, Institute of Physics, A. Mickiewicz University, 60-780 Poznań, Poland
}

(Reçu le 14 juin 1982, accepté le 3 septembre 1982)

\begin{abstract}
Résumé. - L'auteur présente une analyse théorique détaillée de la diffusion lumineuse coopérative, due aux variations des polarisabilités de molécules dissemblables, anisotropes dans leur état fondamental. Les variations des polarisabilités prises en considération sont celles qui sont induites par les fluctuations des champs électriques dipolaires à longue distance dans des régions d'ordre proche. Des calculs complets ont été faits jusqu'au deuxième ordre des variations des polarisabilités en tenant compte des contributions pures et croisées aux constantes de diffusion isotrope et anisotrope. Ces contributions, liées aux interactions binaires ainsi que ternaires et quaternaires, ont été calculées en supposant que les molécules sont en corrélation radiale mais statistiquement indépendantes quant à leurs orientations.
\end{abstract}

\begin{abstract}
A detailed theoretical analysis is given of cooperative light scattering, due to changes in polarizability of unlike molecules, anisotropic in their ground state. The changes in molecular polarizabilities considered are those induced by fluctuations of long-range electric dipolar fields in regions of near ordering. Complete calculations are performed with accuracy to the second order of changes in polarizability taking into account pure as well as cross contributions to the constants of isotropic and anisotropic light scattering. The contributions in question are related with two-body as well as three- and four-body interactions, on the specific assumption that the molecules are correlated radially but statistically independent orientationally.
\end{abstract}

1. Introduction. - To Smoluchowski [1] and Einstein [2] is due the concept that spontaneous fluctuations in density and concentration of the scattering system are essential in the process of Rayleigh scattering. Equally fertile was the idea put forward by Yvon [3] concerning the role, in propagation and scattering of light, of statistical translational fluctuations of atoms, correlated by dipolar forces into two- and three-body assemblages. The Yvon model of dipole-induced dipole interaction (DID) was subsequently extended to the case of intrinsically anisotropic molecules and applied to calculate the LorentzLorenz function (or molecular optical refraction) $[4,5]$ as well as the second virial coefficient of isotropic and anisotropic light scattering by compressed gases $[6,7]$.

The depolarization of light due to changes in polarizability of anisotropic molecules in condensed gas mixtures has also been analysed $[8,9]$, applying two

(*) This research was supported by the Polish Academy of Sciences (Research Project MR I.9). distinct molecular models : (i) the S-model (specific model), considering the anisotropic molecules as correlated radially but having statistically independent orientations $[4,5,7,8]$, and (ii) the (general) G-model, considering both the positions and orientations of the anisotropic molecules as statistically dependent [9-13]. On the latter G-model, analytical and numerical calculations have been successfully carried out to the end for the radial-angular correlations with regard to the two limiting cases of (i) weak angular (tensorial) interactions, dealt with as a perturbation to the radial interactions $[6,8,11]$, and (ii) strong angular interactions, expressed in terms of two-parameter Langevin functions [12-14].

In addition to the preceding integral scattering processes, recent years have witnessed the development of spectral studies of light scattering, particularly with regard to the depolarized component. In their theoretical description of the spectral distribution of light scattered by anisotropic molecules, some authors [15-22] have applied Yvon's model of variable polarizability in the DID approximation. The final 
results, however, were by no means always complete or correct because of the unjustified restrictions imposed in certain cases. The source of the inconsistencies lies in the fact that, in the effective polarizability tensor $\mathbf{A}=\mathbf{A}_{0}+\delta \mathbf{A}$ (with $\mathbf{A}_{0}$ - the molecular polarizability in the absence of interactions), various authors give different interpretations to the change in polarizability tensor $\delta \mathbf{A}$ (caused by molecular interactions or other stochastical factors). As is well known, the intensity of integral light scattering is proportional to the statistical average

$$
\begin{aligned}
\left\langle|\mathbf{A}|^{2}\right\rangle= & \left\langle\left|\mathbf{A}_{0}\right|^{2}\right\rangle+ \\
& +\left\langle\mathbf{A}_{0}^{*} \delta \mathbf{A}+\delta \mathbf{A}^{*} \mathbf{A}_{0}\right\rangle+\left\langle|\delta \mathbf{A}|^{2}\right\rangle,
\end{aligned}
$$

where the first term determines scattering by molecules having unchanged polarizability $\mathbf{A}_{0}$ whereas the higher terms determine coherent scattering on mutually correlated molecules with an increment of polarizability $\delta \mathbf{A}$.

When having recourse to the DID model of Yvon, $\delta \mathbf{A}$ can be calculated in a perturbational approach by writing, with accuracy to the second order $[6,8]$ : $\delta \mathbf{A}=\mathbf{A}_{1}+\mathbf{A}_{2}+\cdots\left(A_{1}, A_{2}\right.$ are defined in $\left.\S 4\right)$. Earlier [6-9] and, in particular, recent papers [14-18] are restricted to calculations of the first order perturbations $\mathbf{A}_{1}$. This, however, may lack justification when applying the G-model since, here, the crossterm contributions $\left\langle\mathbf{A}_{0}^{*} \mathbf{A}_{1}+\mathbf{A}_{1}^{*} \mathbf{A}_{0}\right\rangle$ can be nonzero $[6,9,11-14]$. These cross-contributions vanish always for the molecular S-model, the only non-zero one being the purely fluctuational contribution $\left\langle\left|\mathbf{A}_{1}\right|^{2}\right\rangle[6,8,11]$ usually taken into account in the spectral theories [15-20]. But it should be kept in mind that contributions of the same order as $\left\langle\left|\mathbf{A}_{1}\right|^{2}\right\rangle$ give non-zero cross-terms $[7,8,11,13]$ $\left\langle\mathbf{A}_{0}^{*} \mathbf{A}_{2}+\mathbf{A}_{2}^{*} \mathbf{A}_{0}\right\rangle$ in the S-model. It is noteworthy that, in the molecular S-model, the factor causing the Lorentz-Lorenz function to be density-dependent is precisely the second-order change in molecular polarizability $\left\langle\mathbf{A}_{2}\right\rangle[4,5]$, whereas the first-approximation variation $\left\langle\mathbf{A}_{1}\right\rangle$ gives non-zero contributions but in the G-model $[5,23,24]$.

It is our aim here to perform to-the-end calculations of integral light scattering on anisotropic molecules with variable polarizability in the DID approximation. We shall show that, in the case of the molecular S-model, terms of the type $\left\langle\mathbf{A}_{0}^{*} \mathbf{A}_{2}+\left|\mathbf{A}_{1}\right|^{2}+\mathbf{A}_{2}^{*} \mathbf{A}_{0}\right\rangle$ give non-zero contributions to coherent light scattering of the isotropic and anisotropic kinds. Similarly, we shall prove that, for the molecular G-model, the cross-terms $\left\langle A_{0}^{*} A_{1}+A_{1}^{*} A_{0}\right\rangle$ already give nonzero contributions to coherent scattering. Our calculations will be carried out for dipolar molecules of arbitrary symmetry applying the formalism of irreducible Cartesian tensors and their unweighted rotational averages [25-27].

2. Light intensity tensors of isotropic and anisotropic scattering. - We consider an isotropic medium of volume $V$, containing a great number $N$ of unlike molecules, intrinsically anisotropic in their ground state. A light wave of electric vector $\mathbf{E}(t)=\mathbf{E}(\omega) \mathrm{e}^{-i \omega t}$ and circular frequency $\omega$ is incident on the medium. Restricting our considerations to the linear response, we can write the electric dipole moment induced in $V$ (neglecting spatial variations of the electric field) as :

$$
M_{\sigma}(t)=A_{\sigma \tau}(\omega) E_{\imath}(t)
$$

where summation extends over the index $\tau$ in accordance with the Einstein convention.

In the absence of electron dispersion and absorption, the tensor of linear dipole polarizability of the volume $V$ can, in a satisfactory approximation, be dealt with as completely symmetric, $A_{\sigma \tau}=A_{\tau \sigma}$, and decomposed into an isotropic and an anisotropic part :

$$
A_{\sigma \tau}=\frac{1}{3} A_{v v} \delta_{\sigma \imath}+D_{\sigma \imath}
$$

Above, $\delta_{\sigma \tau}$ is the symmetric Kronecker tensor. The anisotropic part of the polarizability tensor is given by the deviation tensor

$$
D_{\sigma \tau}=A_{\sigma \tau}-\frac{1}{3} A_{v v} \delta_{\sigma \tau},
$$

the trace of which vanishes, $D_{\sigma \sigma}=0$.

Furthermore, restricting ourselves to electric dipole scattering in the wave zone $\left({ }^{1}\right)$ we obtain by (1) and (2) for the intensity tensors of isotropically and anisotropically scattered light $[22,28]$ :

$$
\begin{gathered}
I_{\sigma \tau}^{\mathrm{is}}=\frac{1}{6}\left(\frac{\omega_{\mathrm{s}}}{c}\right)^{4} L^{\omega_{\mathrm{s}}} S_{\mathrm{is}} E_{\sigma}^{*}(t) E_{\imath}(t), \\
I_{\sigma \tau}^{\mathrm{anis}}=\frac{1}{60}\left(\frac{\omega_{\mathrm{s}}}{c}\right)^{4} L^{\omega_{\mathrm{s}}} S_{\mathrm{anis}}\left\{3|\mathbf{E}(t)|^{2} \delta_{\sigma \imath}+\right. \\
\left.+3 E_{\sigma}(t) E_{\tau}^{*}(t)-2 E_{\sigma}^{*}(t) E_{\imath}(t)\right\},
\end{gathered}
$$

where $L^{\omega_{\mathrm{s}}}$ is a phenomenological parameter, dependent on the geometry of observation and the shape of the scattering specimen. If $V$ is a sphere with the electric permittivity $\varepsilon$ and if observation is carried out in the surrounding medium of permittivity $\varepsilon_{0}$, we have :

$$
L^{\omega_{s}}=\left(\varepsilon_{\omega_{s}}+2 \varepsilon_{0}\right)^{2}\left(\varepsilon_{\omega}+2 \varepsilon_{0}\right)^{2} / 81 \varepsilon_{0}^{4},
$$

where $\varepsilon_{\omega}$ and $\varepsilon_{\omega_{\mathbf{s}}}$ are the electric permittivities for the vibration frequencies $\omega$ and $\omega_{\mathrm{s}}$ of incident and scattered light, respectively.

Equations (3) and (4) contain constants accounting for the molecular-statistical mechanism of isotropic

( $\left.{ }^{1}\right)$ The wave zone of the radiation field is here understood as in classical electrodynamics (see e.g. W. Heitler, The Quantum Theory of Radiation, Oxford, 1954). 
and anisotropic light scattering $\left(^{2}\right)$ (we omit phase interference processes) :

$$
\begin{aligned}
S_{\mathrm{is}} & =\frac{1}{3}\left\langle A_{\sigma \sigma}^{*} A_{\mathfrak{}}\right\rangle, \\
S_{\mathrm{anis}} & =\left\langle D_{\sigma \tau}^{*} D_{\sigma \imath}\right\rangle,
\end{aligned}
$$

with $\langle>$ denoting appropriate statistical averaging at zero external field.

3. The scattering constants in the zeroth approximation. - The problem to be solved now is this : how is the tensor of total polarizability $A_{\sigma \tau}$ of the scattering sample $V$ to be expressed in terms of the properties of the individual molecules or atoms ? Obviously, in the zeroth approximation, $A_{\sigma \tau}$ is the sum of the polarizabilities of the $N$ individual molecules :

$$
A_{\sigma \tau}^{(0)}=\sum_{i=1}^{N} a_{\sigma \imath}^{(i)},
$$

Above, $a_{\sigma \tau}^{(i)}$ is the linear polarizability tensor of the $i$-th isolated molecule.

In the zeroth approximation (8), the scattering constants (6) and (7) lead to results well known in the literature [27]. Nonetheless, we shall consider this simple case in brief to illustrate the course of our further procedure. In particular, with regard to (8), the isotropic scattering constant (6) can be expressed as follows :

$$
S_{\mathrm{is}}^{(0)}=3\left\langle\sum_{i=1}^{N}\left|a_{i}\right|^{2}\right\rangle+3\left\langle\sum_{i=1}^{N} \sum_{j \neq i}^{N} a_{i}^{*} a_{j}\right\rangle,
$$

where

$$
a_{i}=a_{\alpha \alpha}^{(i)} / 3=\left(a_{11}^{(i)}+a_{22}^{(i)}+a_{33}^{(i)}\right) / 3
$$

is the mean polarizability of the $i$-th molecule. The first term of $(6 a)$ determines incoherent isotropic scattering by noninteracting molecules. The second term $(j \neq i)$ describes coherent isotropic scattering by radially correlated molecules.

Before dealing with the anisotropic scattering constant (7) as done for $(6 a)$, we note that the polarizability tensor $a_{\sigma \tau}^{(i)}$ of equation (8) is expressed in the system of laboratory coordinates and that, for the sake of convenience, it should be transformed to a tensor $a_{\alpha \beta}^{(i)}$, expressed in coordinates attached to the molecule :

$$
a_{\sigma \tau}^{(i)}=R_{\sigma \alpha}^{(i)} R_{\tau \beta}^{(i)} a_{\alpha \beta}^{(i)} .
$$

The $R_{\sigma \alpha}^{(i)}$ are rotational transformation coefficients, relating the reference systems $(\sigma, \tau)$ and $(\alpha, \beta)$.

With regard to the transformation (9), the devia-

( $\left.{ }^{2}\right)$ The scattering constants (6) and (7) defined above are, respectively, 3 and 30 times greater than those defined in reference [28] and contain no density fluctuational contributions, which are highly essential for isotropic scattering only. tion tensor $(2 a)$ takes the following form in the zeroth approximation (8) :

$$
D_{\sigma \tau}^{(0)}=\sum_{i=1}^{N}\left(a_{\sigma \tau}^{(i)}-a_{i} \delta_{\sigma \tau}\right)=\sum_{i=1}^{N} R_{\sigma \alpha}^{(i)} R_{\imath \beta}^{(i)} d_{\alpha \beta}^{(i)},
$$

where we have introduced the following deviator of the polarizability tensor in the molecular basis :

$$
d_{\alpha \beta}^{(i)}=a_{\alpha \beta}^{(i)}-a_{i} \delta_{\alpha \beta} .
$$

Applying (10), we write the constant (7) in the form of a sum of incoherent $(i=j)$ and coherent $(i \neq j)$ anisotropic scattering of the zeroth approximation :

$$
\begin{aligned}
S_{\mathrm{anis}}^{(0)} & =\frac{2}{3}\left\langle\sum_{i=1}^{N}\left|\gamma_{i}\right|^{2}\right\rangle+ \\
& +\left\langle\sum_{i=1}^{N} \sum_{j \neq i}^{N} d_{\alpha \beta}^{(i)^{*}} d_{\gamma \delta}^{(j)} R_{\sigma \alpha}^{(i)} R_{\tau \beta}^{(i)} R_{\sigma \gamma}^{(j)} R_{\tau \delta}^{(j)}\right\rangle,
\end{aligned}
$$

where we have introduced the following parameter of absolute anisotropy of the molecular polarizability :

$$
\left|\gamma_{i}\right|^{2}=\frac{3}{2} d_{\alpha \beta}^{(i) *} d_{\alpha \beta}^{(i)}=\frac{3}{2}\left(a_{\alpha \beta}^{(i) *} a_{\alpha \beta}^{(i)}-3\left|a_{i}\right|^{2}\right) .
$$

We now apply the molecular S-model to the coherent part of (7a) implying that the orientations of the individual molecules are statistically independent. This leads to the following unweighted rotational averaging :

$$
\begin{array}{r}
\left\langle R_{\sigma \alpha}^{(i)} R_{\tau \beta}^{(i)} R_{\sigma \gamma}^{(j)} R_{\tau \delta}^{(j)}\right\rangle_{\Omega}=\left\langle R_{\sigma \alpha}^{(i)} R_{\tau \beta}^{(i)}\right\rangle_{\Omega_{i}}\left\langle R_{\sigma \gamma}^{(j)} R_{\imath \delta}^{(j)}\right\rangle_{\Omega_{j}}= \\
=\frac{1}{3} \delta_{\alpha \beta} \delta_{\gamma \delta},
\end{array}
$$

since $[26,27]$

$$
\left\langle R_{\sigma \alpha}^{(i)} R_{\tau \beta}^{(i)}\right\rangle_{\Omega_{i}}=\frac{1}{3} \delta_{\sigma \tau} \delta_{\alpha \beta},\left\langle R_{\sigma \gamma}^{(j)} R_{\imath \delta}^{(j)}\right\rangle_{\Omega_{j}}=\frac{1}{3} \delta_{\sigma \tau} \delta_{\gamma \delta} .
$$

This proves that the part of $(7 a)$ corresponding to coherent scattering vanishes and that, with the Smodel, we have but incoherent anisotropic light scattering

$$
{ }_{\text {incoh }} S_{\text {anis }}^{(0)}=\frac{2}{3}\left\langle\sum_{i=1}^{N}\left|\gamma_{i}\right|^{2}\right\rangle,
$$

studies of which permit the direct determination of the absolute polarizability anisotropy (11) of the isolated molecule. Clearly, in the general G-model, because of the presence of mutual correlation of the molecular orientations, the second term of (7a) accounting for coherent scattering is non-zero. It has been discussed by numerous authors for a variety of angular interactions [6-8, 11-13, 29, 30]. Angular correlations of a similar type intervene in Kerr's effect [31-34] as well as in other nonlinear phenomena [27].

4. Effective linear polarizability in the dipole approximation. - The question now arises of whether the 
S-model admits of coherent anisotropic scattering ? The answer, in the affirmative, has been provided by Yvon [3], who showed that, in dense phases, mutual correlations between the atoms lead to variations of their polarizabilities. In this situation the law of additivity of the polarizabilities (8) no longer holds, and one has to proceed to higher approximations. We write, with accuracy up to the second order [8],

$$
A_{\sigma \tau}=A_{\sigma \tau}^{(0)}+A_{\sigma \tau}^{(1)}+A_{\sigma \tau}^{(2)}+\cdots
$$

where $A_{\sigma \tau}^{(1)}, A_{\sigma \tau}^{(2)}, \ldots$ are deviations of the first, second, ... orders from the additivity law (8) due, in dense systems, to collisions (cf. the model of Silberstein [35] as well as Levine and Birnbaum [36]) and various multipolar interactions between the molecules [27, 28].

We shall consider here only changes in polarizability originating in space and time fluctuations of the long range electric fields $\mathbf{F}(\mathbf{r}, t)$. Restricting ourselves henceforth to interactions of the dipole type we can write, in a linear approximation,

$$
M_{\sigma}(t)=\sum_{i=1}^{N} a_{\sigma \tau}^{(i)}\left\{E_{\tau}(t)+F_{\tau}\left(\mathbf{r}_{i}, t\right)\right\}
$$

where we moreover have to deal with the electric field

$$
F_{\tau}\left(\mathbf{r}_{i}, t\right)=-\sum_{k \neq i}^{N} T_{\tau v}^{(i k)} a_{v \rho}^{(k)}\left\{E_{\rho}(t)+F_{\rho}\left(\mathbf{r}_{k}, t\right)\right\}
$$

produced at the centre of molecule $i$ by the distribution of electric charges of all the $(N-1)$ molecules of the medium polarized by the external field $\mathbf{E}(t)$.
In (14), the tensor of dipole-dipole interaction between the molecules $i$ and $k$ separated by a distance $r_{i k}$ is by definition $(i \neq k$ ) (in the absence of phase interference)

$$
T_{\tau v}^{(i k)}=-r_{i k}^{-5}\left(3 r_{i k \tau} r_{i k v}-r_{i k}^{2} \delta_{\tau v}\right) .
$$

The method of successive substitutions, when applied to equations (13) and (14) with (1) and (12), leads to the following first and second order variations of the polarizability tensor [8] :

$$
\begin{aligned}
& A_{\sigma \imath}^{(1)}=-\sum_{i=1}^{N} \sum_{k \neq i}^{N} a_{\sigma \nu}^{(i)} T_{v \rho}^{(i k)} a_{\rho \tau}^{(k)}, \\
& A_{\sigma \tau}^{(2)}=\sum_{i=1}^{N} \sum_{k \neq i}^{N} \sum_{l \neq k \neq i}^{N} a_{\sigma \nu}^{(i)} T_{v \rho}^{(i k)} a_{\rho \lambda}^{(k)} T_{\lambda \mu}^{(k l)} a_{\mu \tau}^{(l)} .
\end{aligned}
$$

In a similar way, one can calculate the contributions to (12) originating in the nonlinear dipolar polarizabilities of the molecules $[6,8,28,27]$.

5. Coherent scattering in the molecular S-model. We shall now analyse one by one the contributions to the scattering constants (6) and (7) obtained in the approximations (8), (15) and (16) using the molecular S-model.

5.1 CROSS-CONTRIBUTIONS OF THE TYPES 01 AND 10. - In the first approximation we have, for the isotropic scattering constant (6),

$$
S_{\mathrm{is}}^{(1)}=S_{\mathrm{is}}^{(01)}+S_{\mathrm{is}}^{(10)},
$$

where, by (8) and (15),

$$
S_{\mathrm{is}}^{(01)}=\frac{1}{3}\left\langle A_{\sigma \sigma}^{(0)^{*}} A_{\tau \tau}^{(1)}\right\rangle=-\frac{1}{3}\left\langle\sum_{i=1}^{N} \sum_{j=1}^{N} \sum_{k \neq j}^{N} a_{\sigma \sigma}^{(i)^{*}} a_{\tau v}^{(j)} a_{\rho \tau}^{(k)} T_{v \rho}^{(j k)}\right\rangle .
$$

On separating the parts related with two-body interactions for $i=j \neq k$ and $i=k \neq j$, we obtain

$$
{ }_{2} S_{\mathrm{is}}^{(01)}=-\left\langle\sum_{i=1}^{N} \sum_{j \neq i}^{N} a_{i}^{*}\left\{a_{\tau v}^{(i)} a_{\rho \tau}^{(j)}+a_{\rho \tau}^{(i)} a_{\tau v}^{(j)}\right\} T_{v \rho}^{(i j)}\right\rangle .
$$

The S-model, when applied to (18a), permits unweighted averaging separately over the orientations of molecules $i$ and $j$ :

$$
\left\langle a_{\tau v}^{(i)} a_{\rho \tau}^{(j)}\right\rangle_{\Omega}=\left\langle a_{\tau v}^{(i)}\right\rangle_{\Omega_{i}}\left\langle a_{\rho \tau}^{(j)}\right\rangle_{\Omega_{j}}=a_{i} a_{j} \delta_{v \rho}
$$

and we find that the binary contribution (18a) vanishes. Similarly, with the S-model, the ternary contribution resulting from (18) vanishes as well.

By $(2 a)$ and (15), the deviator of the polarizability tensor is, in the first approximation,

$$
D_{\sigma \tau}^{(1)}=-\frac{1}{3} \sum_{i=1}^{N} \sum_{k \neq i}^{N}\left\{3 a_{\sigma \nu}^{(i)} a_{\rho \tau}^{(k)}-a_{\lambda \nu}^{(i)} a_{\rho \lambda}^{(k)} \delta_{\sigma \tau}\right\} T_{\nu \rho}^{(i k)}
$$

and, taking into account the deviator of the zeroth approximation (10), we get from (7) the anisotropic scattering constant of the first approximation in the form :

$$
S_{\mathrm{anis}}^{(1)}=S_{\mathrm{anis}}^{(01)}+S_{\mathrm{anis}}^{(10)},
$$


where

$$
S_{\mathrm{anis}}^{(01)}=-\left\langle\sum_{i=1}^{N} \sum_{j=1}^{N} \sum_{k \neq j}^{N}\left\{a_{\sigma i}^{(i) *} a_{\sigma \nu}^{(j)} a_{\rho \imath}^{(k)}-a_{i}^{*} a_{\sigma \nu}^{(j)} a_{\rho \sigma}^{(k)}\right\} T_{v \rho}^{(j k)}\right\rangle
$$

The constant $S_{\text {anis }}^{(10)}$ is determined similarly.

Along similar lines as in the case of (18), we find immediately that in the case of the S-model the binary and ternary contributions inherent in (21) vanish.

The G-model obviously leads to very different results : in this case, the two constants (18) and (21) yield non-vanishing binary, ternary and higher contributions due to the translational-orientational fluctuations $[6,8-14]$.

5.2 PURe CONTRIBUtions OF THE TYPE 11. - With regard to (15), the next contribution to the isotropic scattering constant (6) is of the form :

$$
S_{\mathrm{is}}^{(11)}=\frac{1}{3}\left\langle\sum_{i=1}^{N} \sum_{j=1}^{N} \sum_{k \neq i}^{N} \sum_{l \neq j}^{N} a_{\sigma \nu}^{(i)^{*}} a_{\tau \lambda}^{(j)} a_{\rho \sigma}^{(k)^{*}} a_{\mu \tau}^{(l)} T_{v \rho}^{(i k)} T_{\lambda \mu}^{(j l)}\right\rangle .
$$

On separating the nonzero terms related with two-body interaction for $i=j \neq k=l$ and $i=l \neq j=k$ we get :

$$
{ }_{2} S_{\mathrm{is}}^{(11)}=\frac{1}{3}\left\langle\sum_{i=1}^{N} \sum_{j \neq i}^{N}\left\{a_{\sigma v}^{(i) *} a_{\tau \lambda}^{(i)} a_{\rho \sigma}^{(j)^{*}} a_{\mu \tau}^{(j)}+a_{\sigma v}^{\left(i *^{*}\right.} a_{\mu \tau}^{(i)} a_{\rho \sigma}^{(j)^{*}} a_{\imath \lambda}^{(j)}\right\} T_{v \rho}^{(i j)} T_{\lambda \mu}^{(i j)}\right\rangle
$$

The S-model enables us to write

$$
\left\langle a_{\sigma \nu}^{(i)^{*}} a_{\tau \lambda}^{(i)} a_{\rho \sigma}^{(j)^{*}} a_{\mu \tau}^{(j)}\right\rangle_{\Omega}=\left\langle a_{\sigma \nu}^{(i)^{*}} a_{\tau \lambda}^{(i)}\right\rangle_{\Omega_{i}}\left\langle a_{\rho \sigma}^{(j)^{*}} a_{\mu \tau}^{(j)}\right\rangle_{\Omega_{j}},
$$

where, with regard to the rotational transformation (9), we now have :

$$
\left\langle a_{\sigma \nu}^{(i)^{*}} a_{\tau \lambda}^{(i)}\right\rangle_{\Omega_{i}}=a_{\alpha \beta}^{(i)^{*}} a_{\gamma \delta}^{(i)}\left\langle R_{\sigma \alpha}^{(i)} R_{\nu \beta}^{(i)} R_{\tau \gamma}^{(i)} R_{\lambda \delta}^{(i)}\right\rangle_{\Omega_{i}}
$$

The appropriate unweighted rotational averaging formulae $[26,27]$ together with equations $(10 a)$ and (11) lead to :

$$
\left\langle a_{\sigma v}^{(i)^{*}} a_{\tau \lambda}^{(i)}\right\rangle_{\Omega_{i}}=\left|a_{i}\right|^{2} \delta_{\sigma v} \delta_{\tau \lambda}+\frac{1}{45}\left|\gamma_{i}\right|^{2}\left(3 \delta_{\sigma \tau} \delta_{v \lambda}+3 \delta_{\sigma \lambda} \delta_{v \tau}-2 \delta_{\sigma v} \delta_{\imath \lambda}\right)
$$

whence we finally obtain :

$$
\begin{aligned}
\left\langle a_{\sigma v}^{(i)^{*}} a_{\imath \lambda}^{(i)}\right\rangle_{\Omega_{i}}\left\langle a_{\rho \sigma}^{(j)^{*}} a_{\mu \nu}^{(j)}\right\rangle_{\Omega_{j}}=\left|a_{i}\right|^{2}\left|a_{j}\right|^{2} \delta_{v \rho} & \delta_{\lambda \mu}+ \\
& +\frac{1}{45}\left(\left|a_{i}\right|^{2}\left|\gamma_{j}\right|^{2}+\left|a_{j}\right|^{2}\left|\gamma_{i}\right|^{2}\right)\left(3 \delta_{v \lambda} \delta_{\rho \mu}+3 \delta_{v \mu} \delta_{\rho \lambda}-2 \delta_{v \rho} \delta_{\lambda \mu}\right) \\
& +\frac{1}{2025}\left|\gamma_{i}\right|^{2}\left|\gamma_{j}\right|^{2}\left(33 \delta_{v \lambda} \delta_{\rho \mu}-12 \delta_{v \mu} \delta_{\rho \lambda}+13 \delta_{v \rho} \delta_{\lambda \mu}\right) . \quad(23 a)
\end{aligned}
$$

The above expressions enable us to write the binary contribution (22a) in the final form :

$$
{ }_{2} S_{\mathrm{is}}^{(11)}=\frac{4}{15}\left\langle\sum_{i=1}^{N} \sum_{j \neq i}^{N}\left\{2\left|a_{i}\right|^{2}\left|\gamma_{j}\right|^{2}+2\left|\gamma_{i}\right|^{2}\left|a_{j}\right|^{2}+\frac{7}{45}\left|\gamma_{i}\right|^{2}\left|\gamma_{j}\right|^{2}\right\} r_{i j}^{-6}\right\rangle .
$$

Beside the above two-body interaction contribution we calculate from (22) the three-body interaction contributions for $i=j \neq k \neq l, i=l \neq j \neq k, j=k \neq i \neq l$, and $k=l \neq i \neq j$ :

${ }_{3} S_{i s}^{(11)}=\frac{8}{15}\left\langle\sum_{i=1}^{N} \sum_{j \neq i}^{N} \sum_{k \neq j \neq i}^{N}\left\{a_{i}^{*}\left|\gamma_{j}\right|^{2} a_{k} r_{i j}^{-3} r_{j k}^{-3} P_{2}\left(\frac{\mathbf{r}_{i j} \cdot \mathbf{r}_{j k}}{r_{i j} r_{j k}}\right)+\left|\gamma_{i}\right|^{2} a_{j}^{*} a_{k} r_{i j}^{-3} r_{k i}^{-3} P_{2}\left(\frac{\mathbf{r}_{i j} \cdot \mathbf{r}_{k i}}{r_{i j} r_{j k}}\right)\right\}\right\rangle$,

where $P_{n}$ is a Legendre polynomial of the $n$-th order.

The last remaining term of (22) for $i \neq j \neq k \neq l$ corresponds to four-body interaction. On the S-model it vanishes. In fact, 


$$
{ }_{4} S_{\mathrm{is}}^{(11)}=\frac{1}{3}\left\langle\sum_{i=1}^{N} \sum_{j \neq i}^{N} \sum_{k \neq j \neq i}^{N} \sum_{l \neq k \neq j \neq i}^{N}\left\langle a_{\sigma \nu}^{(i)^{*}}\right\rangle_{\Omega}\left\langle a_{\tau \lambda}^{(j)}\right\rangle_{\Omega}\left\langle a_{\rho \sigma}^{(k) *}\right\rangle_{\Omega}\left\langle a_{\mu \tau}^{(l)}\right\rangle_{\Omega} T_{v \rho}^{(i k)} T_{\lambda \mu}^{(j)}\right\rangle=0,
$$

since $\left\langle a_{\sigma \nu}^{(i) *}\right\rangle_{\Omega}=a_{i}^{*} \delta_{\sigma v}$, etc.

We now proceed to discuss the anisotropic scattering constant (7), for which we have with regard to (19) :

$$
S_{\mathrm{anis}}^{(11)}=\frac{1}{3}\left\langle\sum_{i=1}^{N} \sum_{j=1}^{N} \sum_{k \neq i}^{N} \sum_{l \neq j}^{N} a_{\sigma \nu}^{(i)^{*}}\left\{3 a_{\sigma \lambda}^{(j)} a_{\rho \tau}^{(k)^{*}}-a_{\tau \lambda}^{(j)} a_{\rho \sigma}^{(k)^{*}}\right\} a_{\mu \nu}^{(l)} T_{v \rho}^{(i k)} T_{\lambda \mu}^{(j l)}\right\rangle .
$$

It is essential to note that the anisotropic scattering constant (26) differs from zero even in the case of atoms and isotropically polarizable molecules. In fact, we have here $d_{\alpha \beta}^{(i)}=0$ whence, by (10a),

$$
a_{\alpha \beta}^{(i)}=a_{i} \delta_{\alpha \beta},
$$

and, as a result of the above, $(26)$ gives $[6,8]$ :

$$
S_{\mathrm{anis}}^{(11)}=6\left\langle\sum_{i=1}^{N} \sum_{j=1}^{N} \sum_{k \neq i}^{N} \sum_{l \neq j}^{N} a_{i}^{*} a_{j} a_{k}^{*} a_{l} r_{i k}^{-3} r_{j l}^{-3} P_{2}\left(\frac{\mathbf{r}_{i k} \cdot r_{j l}}{r_{i k} r_{j l}}\right)\right\rangle \text {. }
$$

This, obviously, is the principal result of Yvon's model and derives from the changes in atomic polarizabilities in the electric dipole approximation.

We now apply to (26) the procedure used with regard to the isotropic constant (22). The successive S-model contributions from two-, three- and four-body interactions now are :

$$
\begin{aligned}
& { }_{2} S_{\mathrm{anis}}^{(11)}=6\left\langle\sum_{i=1}^{N} \sum_{j \neq i}^{N}\left\{2\left|a_{i}\right|^{2}\left|a_{j}\right|^{2}+\frac{7}{45}\left(\left|a_{i}\right|^{2}\left|\gamma_{j}\right|^{2}+\left|\gamma_{i}\right|^{2}\left|a_{j}\right|^{2}\right)+\frac{44}{675}\left|\gamma_{i}\right|^{2}\left|\gamma_{j}\right|^{2}\right\} r_{i j}^{-6}\right\rangle, \\
& { }_{3} S_{\text {anis }}^{(11)}=6\left\langle\sum _ { i = 1 } ^ { N } \sum _ { j \neq i } ^ { N } \sum _ { k \neq j \neq i } ^ { N } \left\{ a_{i}^{*}\left(2\left|a_{j}\right|^{2}+\frac{7}{45}\left|\gamma_{j}\right|^{2}\right) a_{k} r_{i j}^{-3} r_{j k}^{-3} P_{2}\left(\frac{\mathbf{r}_{i j} \cdot \mathbf{r}_{j k}}{r_{i j} r_{j k}}\right)+\right.\right. \\
& \left.\left.+\left(2\left|a_{i}\right|^{2}+\frac{7}{45}\left|\gamma_{i}\right|^{2}\right) a_{j}^{*} a_{k} \cdot r_{i j}^{-3} r_{k i}^{-3} P_{2}\left(\frac{\mathbf{r}_{i j} \cdot \mathbf{r}_{k i}}{r_{i j} r_{k i}}\right)\right\}\right\rangle,
\end{aligned}
$$

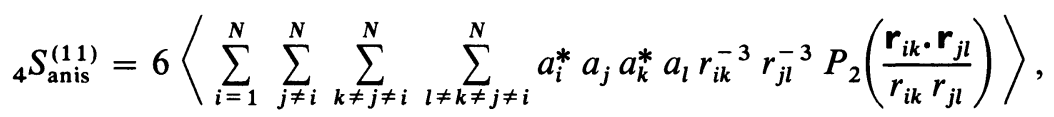

showing once again that in the case of atoms and isotropically polarizable molecules, for which $|\gamma|^{2}=0$, the isotropic scattering constants (24) and (25) vanish whereas the anisotropic constants (28)-(30) go over into the results of Yvon $[3,6,8]$.

5.3 CROSS CONTRIBUTIONS OF THE TYPES 02 AND 20. - We shall now calculate the cross contributions due to interference between polarizabilities of the zeroth order (8) and second-order (16) approximations. In particular, we have for the isotropic scattering constant (6) :

$$
S_{\mathrm{is}}^{(02)}=\frac{1}{3}\left\langle\sum_{i=1}^{N} \sum_{j=1}^{N} \sum_{k \neq j}^{N} \sum_{l \neq k \neq j}^{N} a_{\sigma \sigma}^{\left(i \sigma^{*}\right.} a_{\tau \nu}^{(j)} a_{\rho \lambda}^{(k)} a_{\mu \tau}^{(l)} T_{v \rho}^{(j k)} T_{\lambda \mu}^{(k l)}\right\rangle .
$$

The constant $S_{\text {is }}^{(20)}$ is, obviously, determined similarly.

Prior to discussing in detail the many-body interaction contributions resulting from equation. (31) we draw attention to a general property distinguishing (31) from the previously considered constant (22). Thus, whereas in the case of the isotropic polarizability $(10 b)\left({ }^{3}\right)$ the isotropic scattering constant (22) vanishes immediately irrespective of the molecular model assumed, the constant (31) does not vanish but takes the form :

$$
S_{\mathrm{is}}^{(02)}=6\left\langle\sum_{i=1}^{N} \sum_{j=1}^{N} \sum_{k \neq j}^{N} \sum_{l \neq k \neq j}^{N} a_{i}^{*} a_{j} a_{k} a_{l} r_{k l}^{-3} r_{j k}^{-3} P_{2}\left(\frac{\mathbf{r}_{k l} \cdot \mathbf{r}_{j k}}{r_{k l} r_{j k}}\right)\right\rangle \text {. }
$$

Moreover, this contribution is analogical (though in general not identical) to the contribution (27) for anisotropic scattering. Hence we note that, in the case of atoms and isotropically polarizable molecules, the contri-

$\left({ }^{3}\right)$ Equation $(10 b)$ is to be found just below equation (26). 
bution to isotropic scattering in the 02 and 20 approximations plays a role analogical to that of the contribution to anisotropic scattering in the 11 approximation.

Taking up equation (31), we have for two-body interaction the two components with $i=j=l \neq k$ and $i=k \neq j=l$ which, in the case of the S-model, finally give :

$$
{ }_{2} S_{\mathrm{is}}^{(02)}=6\left\langle\sum_{i=1}^{N} \sum_{j \neq i}^{N}\left\{\left|a_{i}\right|^{2}\left(a_{j}^{2}+\frac{2}{9} \gamma_{j}^{2}\right)+a_{i}^{*} a_{j}\left(a_{i}^{2}+\frac{2}{9} \gamma_{i}^{2}\right)\right\} r_{i j}^{-6}\right\rangle .
$$

Similarly, for three-body interaction $(i=j \neq k \neq l, i=k \neq j \neq l, i=l \neq j \neq k, j=l \neq i \neq k)$, equation (31) leads to :

$$
{ }_{3} S_{\mathrm{is}}^{(02)}=6\left\langle\sum_{i=1}^{N} \sum_{j \neq i}^{N} \sum_{k \neq j \neq i}^{N} a_{i}^{*}\left(a_{j}^{2}+\frac{2}{9} \gamma_{j}^{2}\right) a_{k} r_{j k}^{-6}+\left(a_{i}^{*}+a_{j}^{*}+a_{k}^{*}\right) a_{i} a_{j} a_{k} r_{k l}^{-3} r_{j k}^{-3} P_{2}\left(\frac{\mathbf{r}_{k l} \cdot \boldsymbol{r}_{j k}}{r_{k l} r_{j k}}\right)\right\rangle
$$

and, for four-body interaction $(i \neq j \neq k \neq l)$ :

$$
{ }_{4} S_{\mathrm{is}}^{(02)}=6\left\langle\sum_{i=1}^{N} \sum_{j \neq i}^{N} \sum_{k \neq j \neq i}^{N} \sum_{l \neq k \neq j \neq i}^{N} a_{i}^{*} a_{j} a_{k} a_{l} r_{k l}^{-3} r_{j k}^{-3} P_{2}\left(\frac{\mathbf{r}_{k l} \cdot \mathbf{r}_{j k}}{r_{k l} r_{j k}}\right)\right\rangle .
$$

By (2a) and (16) we obtain the following deviator of the polarizability tensor in the second approximation :

$$
D_{\sigma \tau}^{(2)}=\frac{1}{3} \sum_{i=1}^{N} \sum_{k \neq i}^{N} \sum_{l \neq k \neq i}^{N}\left\{3 a_{\sigma \nu}^{(i)} a_{\mu \tau}^{(l)}-a_{\varepsilon v}^{(i)} a_{\mu \varepsilon}^{(l)} \delta_{\sigma \tau}\right\} a_{\rho \lambda}^{(k)} T_{v \rho}^{(i k)} T_{\lambda \mu}^{(k l)},
$$

and the anisotropic scattering constant (7) becomes :

$$
S_{\mathrm{anis}}^{(02)}=\frac{1}{3}\left\langle\sum_{i=1}^{N} \sum_{j=1}^{N} \sum_{k \neq j}^{N} \sum_{l \neq k \neq j}^{N}\left\{3 a_{\sigma \tau}^{(i)^{*}} a_{\sigma \nu}^{(j)}-a_{\sigma \sigma}^{(i)^{*}} a_{\tau v}^{(j)}\right\} a_{\rho \lambda}^{(k)} a_{\mu \nu}^{(l)} T_{v \rho}^{(j k)} T_{\lambda \mu}^{(k l)}\right\rangle .
$$

In this context it should be noted that by $(10 b)$ for atoms and isotropically polarizable molecules the cross contribution (36) vanishes directly, signifying that in this 02 approximation the Yvon model changes in polarizability produce no anisotropic scattering of light, as it was the case in the 11 approximation, where anisotropic scattering does exist and is always given by (27).

We shall now show that the anisotropic scattering constant (36) can differ from zero provided that the molecules are intrinsically anisotropic in their ground state. In particular, for two-body interaction, equation (36) contains two non-zero terms for $i=j=l \neq k$ and $i=k \neq j=l$ which, using the S-model, we write as follows :

$$
\begin{aligned}
{ }_{2} S_{\text {anis }}^{(02)}=\frac{1}{3}\left\langle\sum _ { i = 1 } ^ { N } \sum _ { j \neq i } ^ { N } \left\{\left\langle\left(3 a_{\sigma \tau}^{\left(i *^{*}\right.} a_{\sigma \nu}^{(i)}-a_{\sigma \sigma}^{(i) *} a_{\tau \nu}^{(i)}\right) a_{\mu \tau}^{(i)}\right\rangle_{\Omega_{i}}\left\langle a_{\rho \lambda}^{(j)}\right\rangle_{\Omega_{j}}+\right.\right. \\
\left.\left.+\left(3\left\langle a_{\sigma \tau}^{(i) *} a_{\rho \lambda}^{(i)}\right\rangle_{\Omega_{i}}\left\langle a_{\sigma \nu}^{(j)} a_{\tau \mu}^{(j)}\right\rangle_{\Omega_{j}}-\left\langle a_{\sigma \sigma}^{(i)^{*}} a_{\rho \lambda}^{(i)}\right\rangle_{\Omega_{i}}\left\langle a_{\tau \nu}^{(j)} a_{\tau \mu}^{(j)}\right\rangle_{\Omega_{J}}\right)\right\} T_{v \rho}^{(i j)} T_{\lambda \mu}^{(i j)}\right\rangle .
\end{aligned}
$$

Applying the rotational average (23) and the following :

$$
\left\langle a_{\sigma \tau}^{(i)^{*}} a_{\sigma \nu}^{(i)} a_{\mu \tau}^{(i)}\right\rangle_{\Omega_{i}}=\frac{1}{3} a_{\alpha \beta}^{(i) *} a_{\alpha \gamma}^{(i)} a_{\gamma \beta}^{(i)} \delta_{v \mu}
$$

we reduce $(36 a)$ to :

$$
{ }_{2} S_{\mathrm{anis}}^{(02)}=\frac{2}{15}\left\langle\sum_{i=1}^{N} \sum_{j \neq i}^{N}\left\{\left(20 a_{i}+a_{j}\right)\left|\gamma_{i}\right|^{2} a_{j}+\frac{10}{3}\left|\gamma_{i}\right|^{3} a_{j}+\frac{1}{45}\left|\gamma_{i}\right|^{2} \gamma_{j}^{2}\right\} r_{i j}^{-6}\right\rangle,
$$

where for simplicity we have used the notation :

$$
\left|\gamma_{i}\right|^{3}=\frac{9}{2} d_{\alpha \beta}^{(i)} d_{\alpha \gamma}^{(i)} d_{\gamma \beta}^{(i)}
$$

On separating in (36) the terms corresponding to three-body interaction, we obtain for the S-model

$$
{ }_{3} S_{\mathrm{anis}}^{(02)}=\frac{2}{15}\left\langle\sum_{i=1}^{N} \sum_{j \neq i}^{N} \sum_{k \neq j \neq i}^{N}\left\{a_{i}\left|\gamma_{j}\right|^{2}+20\left|\gamma_{i}\right|^{2} a_{j}\right\} a_{k} r_{i j}^{-3} r_{j k}^{-3} P_{2}\left(\frac{\mathbf{r}_{i j} \cdot \mathbf{r}_{j k}}{r_{i j} r_{j k}}\right)\right\rangle .
$$


Obviously, the four-body interaction term inherent in (36) vanishes in the case of the S-model :

$$
{ }_{4} S_{\text {anis }}^{(02)}=0 \text {. }
$$

On summation of (28) and (37) as well as (29) and (39) we obtain for $S_{\text {anis }}^{(02)}+S_{\text {anis }}^{(11)}+S_{\text {anis }}^{(20)}$ results for two- and three-body interaction in the form proposed by us in an earlier paper [8] (Eqs. (A. 7)-(A. 10)).

6. Applications and discussion. - The expressions derived in section 5 for the constants of isotropic and anisotropic scattering of light are valid for arbitrary symmetry of the molecules. Their application to atoms and isotropically polarizable molecules has been discussed above. In order to apply them to the comprehensive class of axially symmetric molecules (point groups $C_{\infty v}$ and $D_{\infty h}$, as well as $C_{3 v}, D_{3 h}, D_{3 d}, C_{6 h}$, $\mathrm{D}_{6 \mathrm{~h}}$, etc.) one has to put for the molecular parameters (6b), (11) and (38) :

$$
\begin{aligned}
a_{i} & =\left(a_{\|}^{(i)}+2 a_{\perp}^{(i)}\right) / 3 \\
\left|\gamma_{i}\right|^{2} & =\left|a_{\|}^{(i)}-a_{\perp}^{(i)}\right|^{2}, \\
\left|\gamma_{i}\right|^{3} & =\left|a_{\|}^{(i)}-a_{\perp}^{(i)}\right|^{2}\left(a_{\|}^{(i)}-a_{\perp}^{(i)}\right) .
\end{aligned}
$$

Likewise, the molecular parameters (11) and (8) can be applied to other molecular symmetries, of the point groups $C_{2 v}, D_{2}, D_{2}, D_{2 h}$ as well as $C_{s}, C_{2}, C_{2 h}$.

Equation (28), when applied to axially-symmetric molecules with identical optical properties, goes over into the result of Berrue et al. [7]. Similarly, the expressions (24) and (25) as well as (28)-(30), if applied to axially-symmetric molecules having identical real polarizabilities $a=a_{i}=a_{j}=a_{k}$ and anisotropies $\gamma=\gamma_{i}=\gamma_{j}=a_{\|}-a_{\perp}$, go over into the results recently obtained by Cox and Madden [18] by the method of spherical tensors (albeit with their numerical coefficient at $\gamma^{4}$ incorrect). Our formulae oreover differ from the results of Trappeniers et al. [37], derived on the hypothesis that the orientation and position of the molecule are uncorrelated. However, the work of all above authors does not comprise the contributions of the type 02 and 20 , given by equations (33)-(39).

The complete contributions to the isotropic and anisotropic scattering constants calculated in section 5 are valid not only for molecules of one species but, as well, for mixtures of unlike atoms and mole- cules. The radial parameters of binary and ternary correlations, $\left\langle r_{i j}^{-6}\right\rangle$ and $\left\langle r_{i j}^{-3} r_{j k}^{-3} P_{2}\left(\frac{\mathbf{r}_{i j} \cdot \mathbf{r}_{j k}}{r_{i j} r_{j k}}\right)\right\rangle$ respectively, are accessible to numerical calculation for specific models of radial interaction [13, 27, 38-43]. On performing the numerical calculations for the polar liquids considered in references [11-13], we find that reasonably good agreement with experiment is achieved when taking into account simultaneously all the binary and ternary contributions calculated in section 5 .

On the other hand, the isotropic and anisotropic scattering constants (6) and (7) can be determined numerically from the experimental data. In particular we have by equations (3) and (4) for the vertical and horizontal scattered intensity components

$$
\begin{aligned}
& I_{\mathrm{VV}}^{\mathrm{S}}=\frac{1}{3}\left(\frac{\omega_{\mathrm{s}}}{c}\right)^{4} L^{\omega_{\mathrm{s}}}\left(S_{\mathrm{is}}+\frac{2}{5} S_{\mathrm{anis}}\right) I_{\mathrm{V}}, \\
& I_{\mathrm{HV}}^{\mathrm{S}}=\frac{1}{10}\left(\frac{\omega_{\mathrm{s}}}{c}\right)^{4} L^{\omega_{\mathrm{s}}} S_{\mathrm{anis}} I_{\mathrm{V}},
\end{aligned}
$$

if the incident light is polarized vertically of intensity $I_{\mathrm{v}}$.

Equations (40) and (41) yield for a medium of arbitrary density

$$
\frac{S_{\text {anis }}}{S_{\text {is }}}=\frac{10 D_{\mathrm{v}}}{3-4 D_{\mathrm{v}}}
$$

a relation whose left hand side coincides with the theoretical definition of the optical anisotropy of the medium, and whose right hand side makes it possible to determine the latter directly from experimental data on the depolarization ratio $D_{\mathrm{V}}=I_{\mathrm{HV}}^{\mathrm{S}} / I_{\mathrm{V}}^{\mathrm{S}}$, for incident light polarized vertically.

Similarly, we obtain from (3) and (4) relations at other conditions of observation, namely

$$
\frac{S_{\mathrm{anis}}}{S_{\mathrm{is}}}=\frac{10 R_{+}}{6-5 R_{+}}
$$

where $R_{+}=I_{-+}^{\mathrm{s}} / I_{++}^{\mathrm{s}}$ is the reversal ratio for rightcircularly polarized incident light [27].

Note added in proof. - Recently, BREUER (Mol. Phys. 45 (1982) 349) has evolved a different version of our theory on the basis of the simple shell structure model of the liquid state leading to numerical results in close agreement with experiment.

\section{References}

[1] Smoluchowski, M., Ann. Phys. 25 (1908) 205.

[2] Einstein, A., Ann. Phys. 33 (1910) 1275.

[3] Yvon, J., La Propagation et la Diffusion de la Lumière, Actualités Scientifiques et Industrielles, No 543 (Herman et Cie, Paris) 1937.

[4] Mazur, P. and Postma, B. J., Physica 25 (1959) 251.

[5] Kielich, S., Physica 28 (1962) 1116.
[6] Kielich, S., Acta Phys. Polonica 19 (1960) 149, 711.

[7] Berrue, J., Chave, A., Dumon, S. and Thibeau, M., J. Physique-Lett. 37 (1976) L-165.

[8] Kielich, S., J. Physique 29 (1968) 619.

[9] KIelich, S., Chem. Phys. Lett. 10 (1971) 516 ; Erratum ibid. 19 (1973) 609. 
[10] Keyes, T., Kivelson, D. and Mc Tague, J. P., J. Chem. Phys. 55 (1971) 4096.

[11] Kielich, S. and Woźniak, S., Acta Phys. Polonica A 45 (1974) 163;

Woźniak, S. and Kielich, S., Acta Phys. Polonica A 52 (1977) 863

[12] Kielich, S., Lalanne, J. R. and Martin, F. B., J. Physique Colloq. 33 (1972) C1-191.

[13] WoźniaK, S. and Kielich, S., J. Physique 36 (1975) 1305.

[14] Berrue, J., Chave, A., Dumon, B. and Thibeau, M., J. Physique 39 (1978) 815 ; Can. J. Phys. 59 (1981) 1510.

[15] Ladanyi, B. M. and Keyes, T., Mol. Phys. 33 (1977) 1063, 1247 ; J. Chem. Phys. 68 (1978) 3217.

[16] Keyes, T. and Ladanyi, B. M., Mol. Phys. 33 (1977) $1099,1271$.

[17] Frenkel, D. and Mc Tague, J. P., J. Chem. Phys. 72 (1980) 2801.

[18] Cox, T. I. and Madden, P. A., Mol. Phys. 39 (1980) 1487.

[19] Bancewicz, T., Acta Phys. Polonica A 56 (1979) 431.

[20] Bancewicz, T. and Kielich, S., J. Chem. Phys. 75 (1981) 107

[21] Dill, J. F., Litowitz, T. A. and Bucaro, J. A., J. Chem. Phys. 62 (1975) 3839.

[22] Kielich, S., Bancewicz, T. and WoźniaK, S., Can. J. Phys. 59 (1981) 1620.

[23] Kielich, S., Mol. Phys. 9 (1965) 549; Acta Phys. Polonica 27 (1965) 305; 28 (1965) 95, 459.

[24] Chen, C. C. and Steele, W. A., J. Chem. Phys. 75 (1981) 383.

[25] Jerphagnon, J., Chemla, D. and Bonneville, R., Adv. Phys. 27 (1978) 609.
[26] Kielich, S., Acta Phys. Polonica 20 (1961) 433.

[27] KIELICH, S., Nonlinear Molecular Optics, Izd. Nauka, Moskva, 1981 (in Russian).

[28] Kielich, S., Optics Commun. 34 (1980) 367.

[29] Kielich, S., J. Chem. Phys. 46 (1967) 4090.

[30] Oksengorn, B., C.R. Hebd. Séan. Acad. Sci. B 282 (1976) 223.

[31] KIELICH, S., Dielectric and related molecular processes (Ed. M. Davies, Chem. Soc. London) Vol. 1 1972, Chapter 7.

[32] Gray, C. G. and Gubbins, K. E., Mol. Phys. 30 (1975) 1481.

[33] HøYe, J. S. and Stell, G., J. Chem. Phys. 66 (1977) 795.

[34] Bataglia, M. R., Cox, T. I. and Madden, P. A., Mol. Phys. 37 (1979) 1413.

[35] Silberstein, L., Philos. Mag. (Ser. 6) 33 (1917) 92, 215, 521

[36] Levine, H. B. and Birnbaum, G., J. Chem. Phys. 55 (1971) 2914.

[37] Trappeniers, N. J., Kuz, V. A. and Ten Seldam, C. A., Physica 57 (1972) 294.

[38] Downs, J., Gubbins, K. E., Murad, S. and Gray, C. G., Mol. Phys. 37 (1979) 129.

[39] Stell, G. and Weiss, J. J., Phys. Rev. A 16 (1977) 757 ; A $21(1980) 645$.

[40] Hayment, A. D. J., Rice, S. A. and Madden, W. G., J. Chem. Phys. 75 (1981) 3033.

[41] Balucani, U. and Vallauri, R., Can. J. Phys. 59 (1981) 1504

[42] Caillol, J. M., Levesque, D. and Weiss, J. J., Mol. Phys. 44 (1981) 733.

[43] Weiss, J. J. and Alder, B. J., Chem. Phys. Lett. 81 (1981) 113. 